Research Article

\title{
Eigenfunction Expansions for the Stokes Flow Operators in the Inverted Oblate Coordinate System
}

\author{
Maria Hadjinicolaou and Eleftherios Protopapas \\ School of Science and Technology, Hellenic Open University, 11 Sahtouri Street, 26222 Patras, Greece \\ Correspondence should be addressed to Maria Hadjinicolaou; hadjinicolaou@eap.gr
}

Received 3 January 2016; Revised 20 March 2016; Accepted 14 April 2016

Academic Editor: Maurizio Brocchini

Copyright ( 2016 M. Hadjinicolaou and E. Protopapas. This is an open access article distributed under the Creative Commons Attribution License, which permits unrestricted use, distribution, and reproduction in any medium, provided the original work is properly cited.

\begin{abstract}
When studying axisymmetric particle fluid flows, a scalar function, $\psi$, is usually employed, which is called a stream function. It serves as a velocity potential and it can be used for the derivation of significant hydrodynamic quantities. The governing equation is a fourth-order partial differential equation; namely, $E^{4} \psi=0$, where $E^{2}$ is the Stokes irrotational operator and $E^{4}=E^{2} \circ E^{2}$ is the Stokes bistream operator. As it is already known, $E^{2} \psi=0$ in some axisymmetric coordinate systems, such as the cylindrical, spherical, and spheroidal ones, separates variables, while in the inverted prolate spheroidal coordinate system, this equation accepts $R$-separable solutions, as it was shown recently by the authors. Notably, the kernel space of the operator $E^{4}$ does not decompose in a similar way, since it accepts separable solutions in cylindrical and spherical system of coordinates, while $E^{4} \psi=0$ semiseparates variables in the spheroidal coordinate systems and it $R$-semiseparates variables in the inverted prolate spheroidal coordinates. In addition to these results, we show in the present work that in the inverted oblate spheroidal coordinates, the equation $E^{\prime 2} \psi=0$ also $R$-separates variables and we derive the eigenfunctions of the Stokes operator in this particular coordinate system. Furthermore, we demonstrate that the equation ${E^{\prime}}^{4} \psi=0 R$-semiseparates variables. Since the generalized eigenfunctions of $E^{\prime 2}$ cannot be obtained in a closed form, we present a methodology through which we can derive the complete set of the generalized eigenfunctions of $E^{\prime 2}$ in the modified inverted oblate spheroidal coordinate system.
\end{abstract}

\section{Introduction}

Stokes flow arises in many problems in physics, medicine, and engineering, where the relative motion between particles and fluid is extensively studied, either theoretically or experimentally. It is defined as the steady axisymmetric flow of an incompressible, viscous fluid, and it is employed in cases where the viscous forces dominate the inertial ones or, equivalently, when the Reynolds number is much less than 1; that is, Re $\ll 1$ [1]. It was first studied by Stokes [2,3] for describing the flow around a spherical particle. Stokes flow is mathematically described through the celebrated system of differential equations governing the velocity and the pressure field [1]. In the axisymmetric cases, however, a scalar function $\psi$, namely, the stream function $\psi$, is employed instead, which satisfies the fourth-order partial differential equation $E^{4} \psi=0$, where $E^{4}=E^{2} \circ E^{2}$ and $E^{2}$ is the Stokes irrotational operator $[1,4]$.
With respect to the direction of the flow, the particles may be considered as being either symmetric, for example, spheres [1,4-6], or axisymmetric, for example, spheroids $[1,5,6]$, or nonsymmetric, for example, ellipsoids $[7,8]$. Since the lack of symmetry is the most common situation in physical problems, our interest focuses on the nonspherical geometries starting with the axisymmetric ones. A solution of Stokes flow in spheroidal coordinates was given implicitly by Sampson early in 1891 [8]. Much later, Payne and Pell [9] derived a solution for the case of a spheroid moving along its axis of symmetry within a quiescent viscous fluid, while Davis [10] studied the sedimentation of axisymmetric particles in shear flows. Lai and Mockros [11] calculated the flow field which is generated by a spheroid executing axial translatory oscillations in an infinite, incompressible, viscous fluid. The motion of ellipsoidal particles immersed in a viscous fluid was the subject of a fundamental work of Jeffery [12], while 
Dorrepall et al. [13] studied Stokes flows about a closed torus. Recently, Srivastava et al. [14] dealt with the problem of steady Stokes flow past a deformed sphere, considering that the uniform stream is along the axis of symmetry or perpendicular to the axis of symmetry.

Among the methods for solving a partial differential equation, there exists the well-known method of separation of variables. Happel and Brenner [1], by applying this method and using an effective ad hoc technique, obtained a solution for the boundary value problem of the axisymmetric viscous flow around a single spheroid with different boundary conditions. Unfortunately, due to the difficulties imposed by the coordinate system, they did not manage to provide a general solution of the governing fourth-order partial differential equation. In 1994, Dassios et al. in [15] derived a complete set of eigenfunctions and generalized eigenfunctions of the Stokes operator $E^{2}$ by introducing the notion of semiseparability. Moreover, they calculated the 0 -eigenspace and the generalized 0 -eigenspace of $E^{2}$ in the spheroidal coordinates, in terms of series expansion of specific combinations of particular kind of Gegenbauer functions [16, 17]. Dassios and Vafeas in [18] rewrote in a more convenient manner the expansions of prolate eigenfunction that were obtained in [15] to eliminate some indeterminacies of their application.

Also, Chwang [19] and Chwang and Wu [20-22] provided solutions for the Stokes flow around slender bodies in unbounded fluids through superposition of hydrodynamic singularities. Moreover, Rallison and Acrivos [23] developed the method of boundary integrals of distributed singularities, while Youngren and Acrivos [24] determined the problem of the slow viscous flow of an unbounded fluid past a single solid particle which is formulated exactly as a system of linear integral equations of the first kind for a distribution of Stokeslets over the particle surface. The use of fundamental singularities for modelling the Stokes flows is the subject of another category of studies and it is quite different from the approach presented in this work.

Numerical studies have also been presented. Pitter et al. [25] investigated the flow past a thin oblate spheroid falling at terminal velocity in an infinite, viscous fluid. Datta and Deo [26] calculated numerically the Stokes flow with slip condition in a deformable sphere and in an oblate spheroid with Kuwabara boundary conditions.

In other studies, the solution obtained by Lamb [27, 28] is used. Lamb in [28] derived the solution for Stokes flow around an ellipsoid. Zhuang et al. [29] proposed a threedimensional fundamental solution in terms of the oblate spheroidal coordinates transforming the solid harmonic functions in Lamb's solution.

Stokes flow in the inverted coordinate systems has been also studied. Hellou [30] presented biharmonic solutions for flow in small Re numbers using geometric inversion in two dimensions. Utilising the outcomes of Dassios et al. [15], we have further expanded our studies to Stokes flow problems around nonconvex bodies, such as the inverted prolate spheroids. The obtained expressions were used to model the blood plasma flow past a red blood cell [31], the sedimentation of a red blood cell [32], and also the blood plasma flow around two aggregated Low Density Lipoproteins [33] and the translation of two aggregated Low Density Lipoproteins within blood plasma [34]. Moreover, the authors in [35, 36] derived the eigenfunctions of the Stokes operator $E^{\prime 2}$ in the modified inverted prolate coordinate system, proving that Stokes operator in this system $R$-separates variables. Specifically, since the generalized eigenfunctions cannot be expressed in a closed form, they developed an algorithm for obtaining every generalized eigenfunction of the kernel of ${E^{\prime}}^{2}$ through recurrence relations.

In the present work, we follow the same methodology we developed in [15] and derive the eigenfunctions of the Stokes operator in the modified inverted oblate coordinate system. We provide an algorithm through which we can calculate the generalized eigenfunctions of the Stokes operator $E^{\prime 2}$, since, again, a closed form expression is not feasible. We utilised the concept of semiseparation and the $R$-separation and we obtained the eigenfunctions of the 0-eigenspace of ${E^{\prime}}^{2}$ expressed as products of Gegenbauer functions divided by the Euclidian distance $r$, while the generalized 0-eigenspace of $E^{\prime 2}$ consisted of combinations of products of Gegenbauer functions divided by $r^{3}$. Thus, we further expand the notion of $R$-separability and the $R$-semiseparability, that were introduced first in $[35,36]$ for the inverted prolate spheroidal system, to the inverted oblate coordinate system, defining appropriately the function $R$.

The structure of this paper is as follows. In Section 2, the mathematical background is presented, while in Section 3 we calculate the eigenfunctions of the 0-eigenspace of $E^{\prime 2}$ and we show the methodology to calculate the generalized 0 -eigenfunctions in the modified inverted oblate system of coordinates. In Section 4, we discuss the obtained results.

\section{Mathematical Background}

Each point $\left(x_{1}, x_{2}, x_{3}\right)$ in Cartesian coordinates can be represented in the oblate spheroidal coordinates $(\lambda, \zeta, \varphi)$ by the equations [37]

$$
\begin{aligned}
& x_{1}=\bar{c} \sqrt{\lambda^{2}+1} \sqrt{1-\zeta^{2}} \cos \varphi, \\
& x_{2}=\bar{c} \sqrt{\lambda^{2}+1} \sqrt{1-\zeta^{2}} \sin \varphi \\
& x_{3}=\bar{c} \lambda \zeta
\end{aligned}
$$

where $\lambda \in \mathbb{R},-1 \leq \zeta \leq 1, \varphi \in[0,2 \pi)$, and $\bar{c}>0$ specifies the semifocal distance.

Consequently,

$$
\begin{aligned}
\mathbf{r}= & \left(\bar{c} \sqrt{\lambda^{2}+1} \sqrt{1-\zeta^{2}} \cos \varphi, \bar{c} \sqrt{\lambda^{2}+1} \sqrt{1-\zeta^{2}}\right. \\
& \cdot \sin \varphi, c \lambda \zeta)
\end{aligned}
$$

with

$$
r=|\mathbf{r}|=\bar{c} \sqrt{\lambda^{2}-\zeta^{2}+1} .
$$


Assuming a sphere of radius $b>0$ with respect to that sphere, the inverted oblate spheroidal coordinates are defined as in [37]

$$
\begin{aligned}
& x_{1}^{\prime}=\frac{b^{2} \sqrt{\lambda^{2}+1} \sqrt{1-\zeta^{2}} \cos \varphi}{\bar{c}\left(\lambda^{2}-\zeta^{2}+1\right)}, \\
& x_{2}^{\prime}=\frac{b^{2} \sqrt{\lambda^{2}+1} \sqrt{1-\zeta^{2}} \sin \varphi}{\bar{c}\left(\lambda^{2}-\zeta^{2}+1\right)}, \\
& x_{3}^{\prime}=\frac{b^{2} \lambda \zeta}{\bar{c}\left(\lambda^{2}-\zeta^{2}+1\right)},
\end{aligned}
$$

where

$$
\begin{aligned}
\mathbf{r}^{\prime} & =\left(\frac{b^{2} \sqrt{\lambda^{2}+1} \sqrt{1-\zeta^{2}} \cos \varphi}{\bar{c}\left(\lambda^{2}-\zeta^{2}+1\right)},\right. \\
& \left.\frac{b^{2} \sqrt{\lambda^{2}+1} \sqrt{1-\zeta^{2}} \sin \varphi}{\bar{c}\left(\lambda^{2}-\zeta^{2}+1\right)}, \frac{b^{2} \lambda \zeta}{\bar{c}\left(\lambda^{2}-\zeta^{2}+1\right)}\right), \\
r^{\prime} & =\left|\mathbf{r}^{\prime}\right|=\frac{b^{2}}{\bar{c} \sqrt{\lambda^{2}-\zeta^{2}+1}} .
\end{aligned}
$$

In what follows, we denote with primes the corresponding quantities in the inverted coordinate system.

\section{R-Separation of the Stokes Operator and $R$-Semiseparation of the Bistream Operator in the Inverted Oblate Coordinate System}

3.1. R-Separation of the Stream Operator in $\Omega^{\prime}$. The Stokes stream operator in the inverted oblate system is

$$
\begin{aligned}
E^{\prime 2} & =\frac{\bar{c}^{2}\left(\lambda^{2}-\zeta^{2}+1\right)}{b^{4}\left(\lambda^{2}+\zeta^{2}\right)}\left\{2 \lambda\left(\lambda^{2}+1\right) \frac{\partial}{\partial \lambda}\right. \\
& +\left(\lambda^{2}+1\right)\left(\lambda^{2}-\zeta^{2}+1\right) \frac{\partial^{2}}{\partial \lambda^{2}}-2 \zeta\left(1-\zeta^{2}\right) \frac{\partial}{\partial \zeta} \\
& \left.+\left(1-\zeta^{2}\right)\left(\lambda^{2}-\zeta^{2}+1\right) \frac{\partial^{2}}{\partial \zeta^{2}}\right\} .
\end{aligned}
$$

which satisfy the equations

$$
E^{\prime 2} \Theta_{n}^{\prime(i)}=0, \quad i=1,2,3,4,
$$$$
\Theta_{n}^{\prime(3)}(\lambda, \zeta)=\frac{b}{\bar{c} \sqrt{\lambda^{2}-\zeta^{2}+1}} H_{n}(i \lambda) G_{n}(\zeta) \text {, }
$$$$
\Theta_{n}^{\prime(4)}(\lambda, \zeta)=\frac{b}{\bar{c} \sqrt{\lambda^{2}-\zeta^{2}+1}} H_{n}(i \lambda) H_{n}(\zeta) \text {, }
$$

so the eigenfunctions $\Theta_{n}^{\prime(i)}(\lambda, \zeta)$ belong in the kernel of the operator $E^{\prime 2}$. Therefore, the Stokes stream operator $R$-separates variables with

$$
R=r
$$

Note that although the argument is a complex number, the Gegenbauer functions $G_{n}$ and $H_{n}$ are real valued. Therefore, a complete representation of the kernel of the operator $E^{\prime 2}$ is given as follows, for any $\psi \in \operatorname{Ker} E^{\prime 2}$ :

$$
\psi(\lambda, \zeta)=\sum_{n=0}^{\infty} \sum_{i=1}^{4} A_{n}^{i} \Theta_{n}^{\prime(i)}(\lambda, \zeta)
$$

where $A_{n}^{i}$ are constants.

3.2. R-Semiseparation of the Bistream Operator in $\Omega^{\prime}$. Following the same methodology we proposed in $[18,19]$, and since, again, a closed form for the generalized eigenfunctions, $\Omega_{n}^{\prime(i)}(\lambda, \zeta)$, cannot be obtained, we derive every $\Omega_{n}^{\prime(i)}(\lambda, \zeta)$ using recurrence relations as follows.

The generalized eigenfunctions from $n=0$ to $n=3$ are

$$
\begin{aligned}
& \Omega_{0}^{\prime(1)}(\lambda, \zeta)=\frac{G_{0}(i \lambda) G_{0}(\zeta)}{6{\sqrt{\lambda^{2}-\zeta^{2}+1}}^{3}}, \\
& \Omega_{1}^{\prime(1)}(\lambda, \zeta)=\frac{G_{1}(i \lambda) G_{1}(\zeta)}{2{\sqrt{\lambda^{2}-\zeta^{2}+1}}^{3}}, \\
& \Omega_{2}^{\prime(1)}(\lambda, \zeta)=\frac{G_{2}(i \lambda) G_{2}(\zeta)}{-2{\sqrt{\lambda^{2}-\zeta^{2}+1}}^{3}}, \\
& \Omega_{3}^{\prime(1)}(\lambda, \zeta)=\frac{G_{3}(i \lambda) G_{3}(\zeta)}{-6{\sqrt{\lambda^{2}-\zeta^{2}+1}}^{3}}, \\
& \Omega_{0}^{\prime(2)}(\lambda, \zeta)=\frac{G_{0}(i \lambda) G_{1}(\zeta)}{6 \sqrt{\lambda^{2}-\zeta^{2}+1}}{ }^{3}
\end{aligned}
$$

$$
\begin{aligned}
& +\frac{G_{0}(i \lambda) G_{3}(\zeta)}{-(3 / 2){\sqrt{\lambda^{2}-\zeta^{2}+1}}^{3}}, \\
\Omega_{1}^{\prime(2)}(\lambda, \zeta)= & \frac{G_{1}(i \lambda) G_{0}(\zeta)}{-2{\sqrt{\lambda^{2}-\zeta^{2}+1}}^{3}} \\
& +\frac{G_{1}(i \lambda) G_{2}(\zeta)}{(3 / 2){\sqrt{\lambda^{2}-\zeta^{2}+1}}^{3}}, \\
\Omega_{2}^{\prime(2)}(\lambda, \zeta)= & \frac{G_{2}(i \lambda) H_{2}(\zeta)}{-2{\sqrt{\lambda^{2}-\zeta^{2}+1}}^{3}}+{\frac{G_{2}}{-3}{\sqrt{\lambda^{2}-\zeta^{2}+1}}^{3}}^{\prime 2} \\
\Omega_{3}^{\prime(2)}(\lambda, \zeta)= & \frac{G_{3}(i \lambda) H_{3}(\zeta)}{-6{\sqrt{\lambda^{2}-\zeta^{2}+1}}^{3}}+\frac{G_{3}(i \lambda) G_{0}(\zeta)}{9{\sqrt{\lambda^{2}-\zeta^{2}+1}}^{3}},
\end{aligned}
$$




$$
\begin{aligned}
& \Omega_{0}^{\prime(3)}(\lambda, \zeta)=\frac{G_{1}(i \lambda) G_{0}(\zeta)}{6{\sqrt{\lambda^{2}-\zeta^{2}+1}}^{3}} \\
& +\frac{G_{3}(i \lambda) G_{0}(\zeta)}{-(3 / 2){\sqrt{\lambda^{2}-\zeta^{2}+1}}^{3}}, \\
& {\Omega^{\prime}}_{1}^{(3)}(\lambda, \zeta)=\frac{G_{0}(i \lambda) G_{1}(\zeta)}{-2{\sqrt{\lambda^{2}-\zeta^{2}+1^{3}}}^{3}} \\
& +\frac{G_{2}(i \lambda) G_{1}(\zeta)}{(3 / 2){\sqrt{\lambda^{2}-\zeta^{2}+1^{3}}}^{3}} \\
& {\Omega^{\prime}}_{2}^{(3)}(\lambda, \zeta)=\frac{H_{2}(i \lambda) G_{2}(\zeta)}{-2{\sqrt{\lambda^{2}-\zeta^{2}+1}}^{3}}+\frac{G_{1}(i \lambda) G_{2}(\zeta)}{-3{\sqrt{\lambda^{2}-\zeta^{2}+1}}^{3}},
\end{aligned}
$$

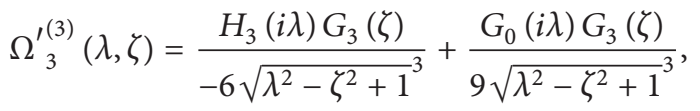

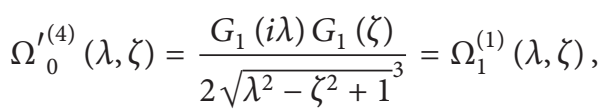

$$
\begin{aligned}
& {\Omega^{\prime}}_{1}^{(4)}(\lambda, \zeta)=\frac{G_{0}(i \lambda) G_{0}(\zeta)}{6{\sqrt{\lambda^{2}-\zeta^{2}+1}}^{3}}=\Omega_{0}^{(1)}(\lambda, \zeta), \\
& {\Omega^{\prime}}_{2}^{(4)}(\lambda, \zeta)=\frac{H_{2}(i \lambda) H_{2}(\zeta)}{-2{\sqrt{\lambda^{2}-\zeta^{2}+1^{3}}}^{3}}+\frac{G_{1}(i \lambda) H_{2}(\zeta)}{-3{\sqrt{\lambda^{2}-\zeta^{2}+1}}^{3}} \\
& +\frac{H_{2}(i \lambda) G_{1}(\zeta)}{-3{\sqrt{\lambda^{2}-\zeta^{2}+1^{3}}}^{3}} \\
& {\Omega^{\prime}}_{3}^{(4)}(\lambda, \zeta)=\frac{H_{3}(i \lambda) H_{3}(\zeta)}{-6{\sqrt{\lambda^{2}-\zeta^{2}+1}}^{3}}+\frac{G_{0}(i \lambda) H_{3}(\zeta)}{9{\sqrt{\lambda^{2}-\zeta^{2}+1}}^{3}} \\
& +\frac{H_{3}(i \lambda) G_{0}(\zeta)}{9{\sqrt{\lambda^{2}-\zeta^{2}+1}}^{3}} \text {. }
\end{aligned}
$$

We also define operator $\Lambda$ as

$$
\begin{aligned}
\Lambda= & 2 \lambda\left(\lambda^{2}+1\right) \frac{\partial}{\partial \lambda}+\left(\lambda^{2}+1\right)\left(\lambda^{2}-\zeta^{2}+1\right) \frac{\partial^{2}}{\partial \lambda^{2}} \\
& -2 \zeta\left(1-\zeta^{2}\right) \frac{\partial}{\partial \zeta}+\left(1-\zeta^{2}\right)\left(\lambda^{2}-\zeta^{2}+1\right) \frac{\partial^{2}}{\partial \zeta^{2}} .
\end{aligned}
$$

Furthermore, we define the functions

$$
\begin{aligned}
\Phi_{2,4}^{(1)} & =\Lambda \frac{G_{2}(i \lambda) G_{2}(\zeta)}{-(8 / 5){\sqrt{\lambda^{2}-\zeta^{2}+1}}^{3}}, \\
\Phi_{3,5}^{(1)} & =\Lambda \frac{G_{3}(i \lambda) G_{3}(\zeta)}{-(24 / 7){\sqrt{\lambda^{2}-\zeta^{2}+1}}^{3}}, \\
\Phi_{2,4}^{(2)} & =\Lambda\left(\frac{G_{2}(i \lambda) H_{2}(\zeta)}{-(8 / 5){\sqrt{\lambda^{2}-\zeta^{2}+1}}^{3}}\right. \\
+ & \left.\frac{G_{2}(i \lambda) G_{1}(\zeta)}{-(72 / 25){\sqrt{\lambda^{2}-\zeta^{2}+1}}^{3}}\right),
\end{aligned}
$$

$$
\begin{aligned}
\Phi_{3,5}^{(2)} & =\Lambda\left(\frac{G_{3}(i \lambda) H_{3}(\zeta)}{-(24 / 7){\sqrt{\lambda^{2}-\zeta^{2}+1}}^{3}}\right. \\
& \left.+\frac{G_{3}(i \lambda) G_{0}(\zeta)}{(360 / 49){\sqrt{\lambda^{2}-\zeta^{2}+1}}^{3}}\right),
\end{aligned}
$$$$
\Phi_{2,4}^{(3)}=\Lambda\left(\frac{H_{2}(i \lambda) G_{2}(\zeta)}{-(8 / 5){\sqrt{\lambda^{2}-\zeta^{2}+1}}^{3}}\right.
$$$$
\left.+\frac{G_{1}(i \lambda) G_{2}(\zeta)}{-(72 / 25){\sqrt{\lambda^{2}-\zeta^{2}+1}}^{3}}\right),
$$$$
\Phi_{3,5}^{(3)}=\Lambda\left(\frac{H_{3}(i \lambda) G_{3}(\zeta)}{-(24 / 7){\sqrt{\lambda^{2}-\zeta^{2}+1}}^{3}}\right.
$$$$
\left.+\frac{G_{0}(i \lambda) G_{3}(\zeta)}{(360 / 49){\sqrt{\lambda^{2}-\zeta^{2}+1}}^{3}}\right),
$$$$
\Phi_{2,4}^{(4)}=\Lambda\left(\frac{H_{2}(i \lambda) H_{2}(\zeta)}{-(8 / 5){\sqrt{\lambda^{2}-\zeta^{2}+1}}^{3}}\right.
$$$$
+\frac{G_{1}(i \lambda) H_{2}(\zeta)}{-(72 / 25){\sqrt{\lambda^{2}-\zeta^{2}+1}}^{3}}
$$$$
\left.+\frac{H_{2}(i \lambda) G_{1}(\zeta)}{-(72 / 25){\sqrt{\lambda^{2}-\zeta^{2}+1}}^{3}}\right),
$$$$
\Phi_{3,5}^{(4)}=\Lambda\left(\frac{H_{3}(i \lambda) H_{3}(\zeta)}{-(24 / 7){\sqrt{\lambda^{2}-\zeta^{2}+1}}^{3}}\right.
$$$$
+\frac{G_{0}(i \lambda) H_{3}(\zeta)}{(360 / 49){\sqrt{\lambda^{2}-\zeta^{2}+1}}^{3}}
$$$$
\left.+\frac{H_{3}(i \lambda) G_{0}(\zeta)}{(360 / 49){\sqrt{\lambda^{2}-\zeta^{2}+1}}^{3}}\right) .
$$

Using the relations

$$
\begin{aligned}
& \Lambda \Omega_{n}^{\prime(i)}=-\alpha_{n} \Phi_{n-2, n}^{(i)}+\beta_{n} \Phi_{n, n+2}^{(i)}, \\
& \Lambda \frac{f_{n}(i \lambda) g_{n}(\zeta)}{{\sqrt{\lambda^{2}-\zeta^{2}+1}}^{3}} \\
& \quad=\frac{1}{{\sqrt{\lambda^{2}-\zeta^{2}+1}}^{3}}\left\{-i 4 \lambda\left(\lambda^{2}+1\right) f_{n}^{\prime}(i \lambda) g_{n}(\zeta)\right. \\
& \quad-\left(\lambda^{2}+1\right)\left(\lambda^{2}-\zeta^{2}+1\right) f_{n}^{\prime \prime}(i \lambda) g_{n}(\zeta) \\
& \quad+6\left(\lambda^{2}+\zeta^{2}\right) f_{n}(i \lambda) g_{n}(\zeta) \\
& \quad-4 \zeta\left(\zeta^{2}-1\right) f_{n}(i \lambda) g_{n}^{\prime}(\zeta) \\
& \left.\quad+\left(\zeta^{2}-1\right)\left(\lambda^{2}-\zeta^{2}+1\right) f_{n}(i \lambda) g_{n}^{\prime \prime}(\zeta)\right\},
\end{aligned}
$$




$$
\begin{aligned}
& \Lambda \frac{f_{n}(i \lambda) g_{n}(\zeta)}{{\sqrt{\lambda^{2}-\zeta^{2}+1}}^{3}}=-(4 n+2) \alpha_{n} \Phi_{n-2, n}^{(i)}-(4 n-6) \\
& \quad \cdot \beta_{n} \Phi_{n, n+2}^{(i)}
\end{aligned}
$$

we can calculate every generalized eigenfunction $\Omega_{n}^{\prime(i)}(\lambda, \zeta)$.

\section{Discussion}

The complete set of the eigenfunctions of the Stokes operator in the modified inverted oblate coordinate system is obtained for the first time. The 0-eigenspace of the operator $E^{\prime 2}$ is given as $R$-separable solutions of Gegenbauer functions of the angular and radial dependence, with $R$ being the Euclidian distance $r$, expressed in the particular coordinate system. The generalized eigenfunctions of the operator $E^{\prime 2}$ in the modified inverted oblate coordinate system are obtained through an algorithmic procedure presented in Section 3. The generalized eigenfunctions are given as the preimages of specific combinations of the 0 -eigenfunctions defined through recurrence relations. They are obtained in $R$-semiseparable form, with $R$ being equal to $r^{3}$ this time. In other words, we demonstrated that the operator $E^{\prime 2} R$-separates variables while the operator $E^{\prime 4}$ " $R$-semiseparates" variables in the modified inverted oblate coordinate system. This "behaviour" reflects the fact of the inversion of the coordinate system and it is closely related to the kind of flow (e.g, irrotational) that the particular Stokes operator represents. The obtained solutions provide a complete decomposition of the 0 -eigenspaces of the Stokes operators in the inverted oblate spheroidal system, allowing each time the choice of the appropriate subspace for representing the solution of the boundary value problem under consideration. As it is already mentioned, the obtained analytical expansions may be used for quantitative and qualitative analysis of problems arising in physics or in medicine, such as blood flow around red blood cells, or the movement of aggregated lipoproteins.

\section{Competing Interests}

The authors declare that they have no competing interests.

\section{Acknowledgments}

This research has been cofinanced by the European Union (European Social Fund, ESF) and Greek national funds through the Operational Program "Education and Lifelong Learning" of the National Strategic Reference Framework (NSRF), Research Funding Program: ARCHIMEDES III. Investing in knowledge society through the European Social Fund.

\section{References}

[1] J. Happel and H. Brenner, Low Reynolds Number Hydrodynamics, Kluwer Academic, New York, NY, USA, 1991.
[2] G. G. Stokes, "On the theories of the internal friction of fluids in motion and the equilibrium and motion of elastic solids," Transactions of the Cambridge Philosophical Society, vol. 8, pp. 287-319, 1945.

[3] G. G. Stokes, "On the effect of the internal friction of fluids on the motion of pendulums," Transactions of the Cambridge Philosophical Society, vol. 8, pp. 8-106, 1851.

[4] H. Brenner, "Rheology of a dilute suspension of axisymmetric Brownian particles," International Journal of Multiphase Flow, vol. 1, no. 2, pp. 195-341, 1974.

[5] S. Kim and J. Karrila, Microhydrodynamics: Principles and Selected Applications, Butterworth-Heinemann, Boston, Mass, USA, 1991.

[6] H. Lamb, Hydrodynamics, Dover, New York, NY, USA, 6th edition, 1945.

[7] A. Oberbeck, "Uber stationare Flussigkeitbewegungen mit Beruecksichtigung der inneren Reiburg," Journal für die Reine und Angewandte Mathematik, vol. 81, pp. 62-80, 1876.

[8] R. A. Sampson, "On stokes current function," Philosophical Transactions of the Royal Society A, vol. 182, pp. 449-518, 1981.

[9] L. E. Payne and W. H. Pell, "The Stokes flow problem for a class of axially symmetric bodies," Journal of Fluid Mechanics, vol. 7, pp. 529-549, 1960.

[10] R. H. Davis, "Sedimentation of axisymmetric particles in shear flows," Physics of Fluids A, vol. 3, no. 9, pp. 2051-2060, 1991.

[11] R. Y. S. Lai and L. F. Mockros, "The Stokes-flow drag on prolate and oblate spheroids during axial translatory accelerations," Journal of Fluid Mechanics, vol. 52, no. 1, pp. 1-15, 1972.

[12] G. B. Jeffery, "The motion of ellipsoidal particles immersed in a viscous fluid," Proceedings of the Royal Society A: Mathematical, Physical and Engineering Sciences, vol. 102, no. 715, pp. 161-179, 1922.

[13] M. J. Dorrepall, R. S. Majumdar, E. M. O’Neil, and B. K. Ranger, "A closed torus in Stokes flow," The Ouarterly Journal of Mechanics and Applied Mathematics, Oxford Journals, vol. 29, no. 4, pp. 381-397, 1975.

[14] K. D. Srivastava, R. R. Yadav, and S. Yadav, "Steady stokes flow around deformed sphere. Class of oblate axisymmetric bodies," International Journal of Applied Mathematics and Mechanics, vol. 8, no. 9, pp. 17-53, 2012.

[15] G. Dassios, M. Hadjinicolaou, and A. C. Payatakes, "Generalized eigenfunctions and complete semiseparable solutions for Stokes flow in Spheroidal Coordinates," Quarterly of Applied Mathematics, vol. 52, no. 1, pp. 157-191, 1994.

[16] N. N. Lebedev, Special Functions and Their Applications, Dover, New York, NY, USA, 1972.

[17] X. Z. Wang and R. D. Guo, Special Functions, World Scientific, 2010.

[18] G. Dassios and P. Vafeas, "On the spheroidal semiseparation for stokes flow," Research Letters in Physics, vol. 2008, Article ID 135289, 4 pages, 2008.

[19] A. T. Chwang, "Hydromechanics of low-reynolds-number flow. Part 3. Motion of a spheroidal particle in quadratic flows," Journal of Fluid Mechanics, vol. 72, no. 1, pp. 17-34, 1975.

[20] A. T. Chwang and Y.-T. Wu, "Hydromechanics of low-Reynoldsnumber flow. Part 1. Rotation of axisymmetric prolate bodies," Journal of Fluid Mechanics, vol. 63, no. 3, pp. 607-622, 1974.

[21] A. T. Chwang and T. Y.-T. Wu, "Hydrodynamics of low reynolds number flow. Part 2. Singularity methods for stokes flows," Journal of Fluid Mechanics, vol. 67, no. 4, pp. 787-815, 1975. 
[22] T. A. Chwang and Y. T. Wu, "Hydrodynamics of low reynolds number flow. Part 4. Translation of spheroids," Journal of Fluid Mechanics, vol. 75, pp. 677-689, 1976.

[23] J. M. Rallison and A. Acrivos, "A numerical study of the deformation and burst of a viscous drop in an extensional flow," Journal of Fluid Mechanics, vol. 89, no. 1, pp. 191-200, 1978.

[24] G. K. Youngren and A. Acrivos, "Stokes flow past a particle of arbitrary shape: a numerical method of solution," Journal of Fluid Mechanics, vol. 69, no. 2, pp. 377-403, 1974.

[25] R. L. Pitter, H. R. Pruppacher, and A. E. Hamielec, "A numerical study of viscous flow past a thin oblate spheroid at low and intermediate reynolds numbers," Journal of the Atmospheric Sciences, vol. 30, no. 1, pp. 125-134, 1973.

[26] S. Datta and S. Deo, "Stokes flow with slip and Kuwabara boundary conditions," Proceedings of the Indian Academy of Sciences-Mathematical Sciences, vol. 112, no. 3, pp. 463-475, 2002.

[27] H. Lamb, "On the conditions for steady motion of a fluid," Proceedings of the London Mathematical Society, vol. 9, article 91, 1978.

[28] H. Lamb, Hydrodynamics, Cambridge University Press, Cambridge, UK, 1932

[29] H. Zhuang, Z. Y. Yan, and W. Y. Wu, “The three-dimensional fundamental solution to Stokes flow in the oblate spheroidal coordinates with applications to multiples spheroid problems," Applied Mathematics and Mechanics, vol. 23, no. 5, pp. 514-534, 2002.

[30] M. Hellou, "Geometric inversion of two-dimensional stokes flows-application to the flow between parallel planes," Engineering, vol. 2, no. 10, pp. 780-787, 2010.

[31] G. Dassios, M. Hadjinicolaou, and E. Protopapas, "Blood plasma flow past a red blood cell: mathematical modelling and analytical treatment," Mathematical Methods in the Applied Sciences, vol. 35, no. 13, pp. 1547-1563, 2012.

[32] M. Hadjinicolaou, G. Kamvyssas, and E. Protopapas, "Stokes flow applied to the sedimentation of a red blood cell," Quarterly of Applied Mathematics, vol. 73, no. 3, pp. 511-523, 2015.

[33] M. Hadjinicolaou, "A mathematical model for the blood plasma flow around two aggregated low-density lipoproteins," in GeNeDis 2014: Computational Biology and Bioinformatics, vol. 820 of Advances in Experimental Medicine and Biology, pp. 173-184, Springer, Berlin, Germany, 2015.

[34] M. Hadjinicolaou and E. Protopapas, "Translation of two aggregated low-density lipoproteins within blood plasma: a mathematical model," in GeNeDis 2014, P. Vlamos and A. Alexiou, Eds., vol. 820 of Advances in Experimental Medicine and Biology, pp. 185-192, Springer, 2015.

[35] M. Hadjinicolaou and E. Protopapas, "On the R-semiseparation of the Stokes bi-stream operator in the inverted prolate spheroidal coordinates," Mathematical Methods in the Applied Sciences, vol. 37, no. 2, pp. 207-211, 2014.

[36] M. Hadjinicolaou and E. Protopapas, "Spectral decomposition of the Stokes flow operators in the inverted prolate spheroidal coordinates," The IMA Journal of Applied Mathematics, vol. 80, no. 5, pp. 1475-1491, 2015.

[37] P. Moon and D. E. Spencer, Field Theory Handbook, Springer, New York, NY, USA, 1961. 


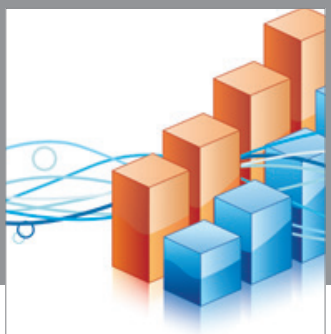

Advances in

Operations Research

vatem alat4

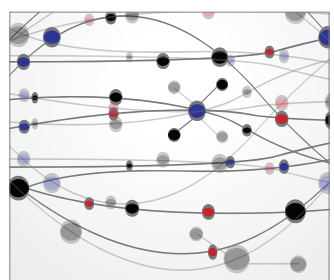

\section{The Scientific} World Journal
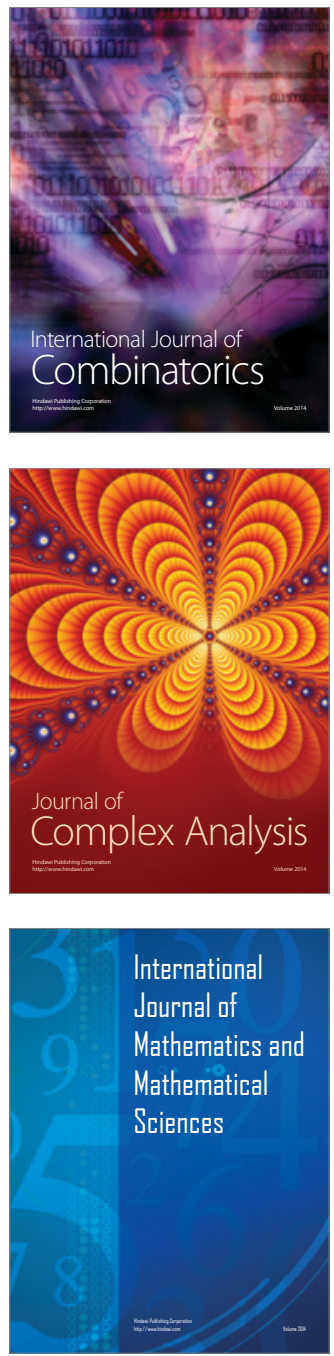
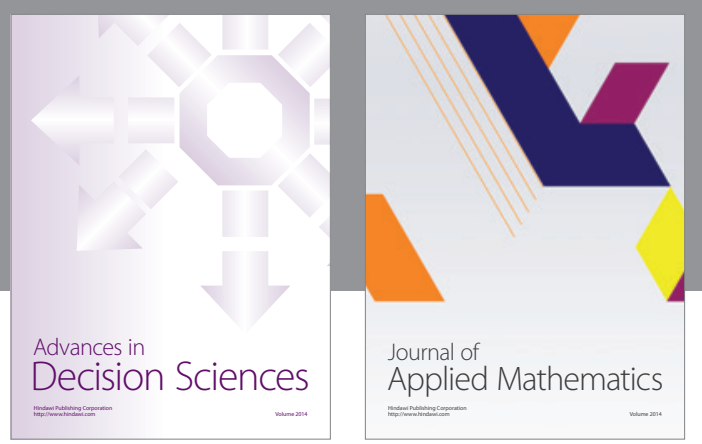

Algebra

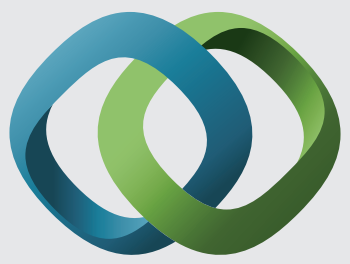

\section{Hindawi}

Submit your manuscripts at

http://www.hindawi.com
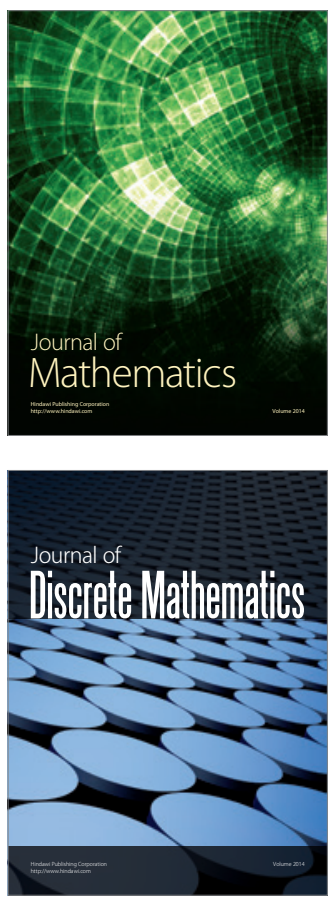

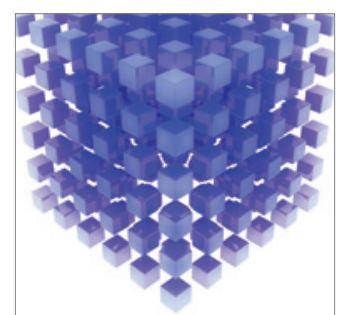

Mathematical Problems in Engineering
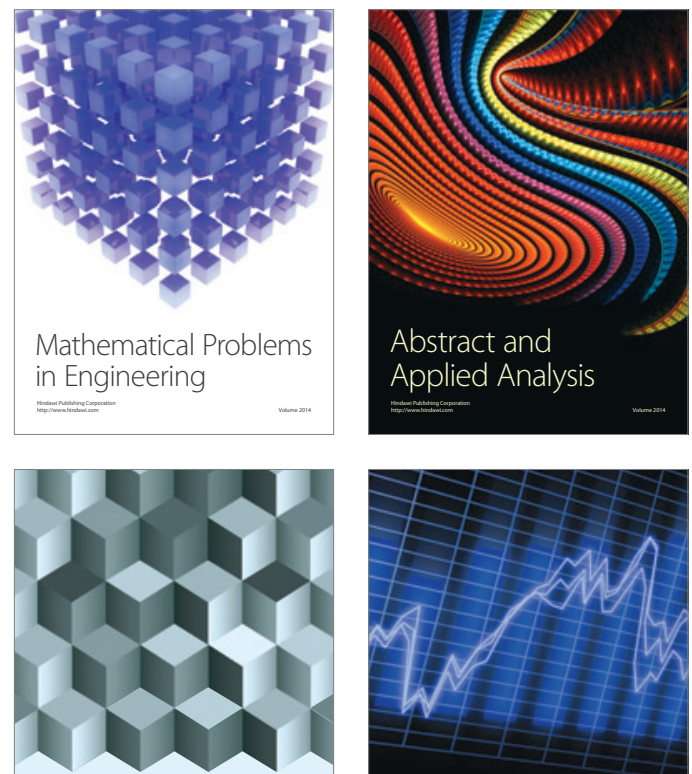

Journal of

Function Spaces

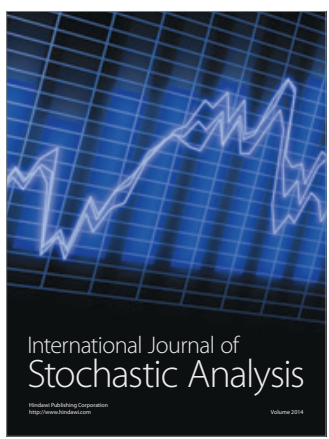

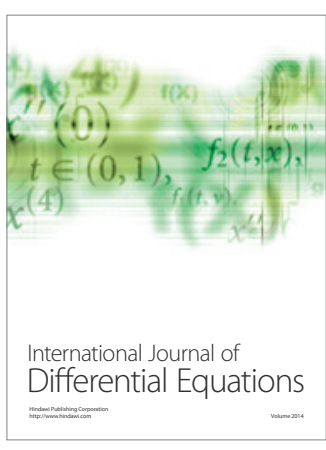
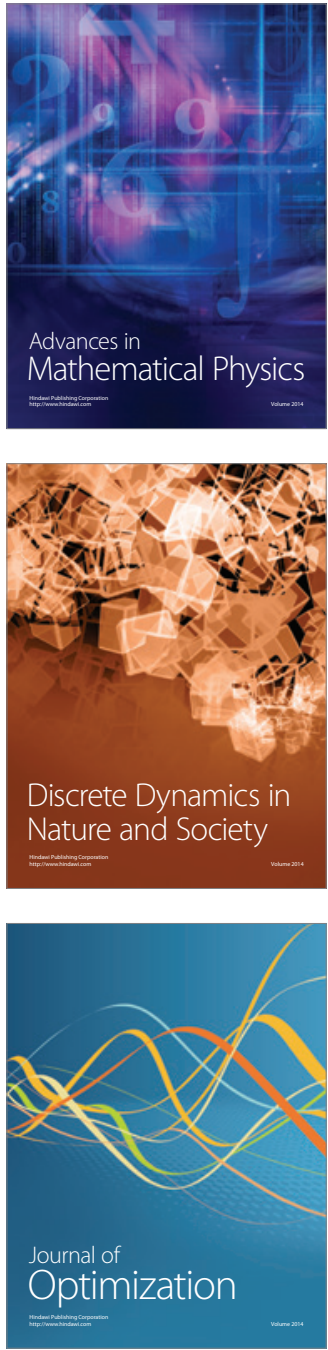